\title{
A Python based Regression Approach on Reliable Journal Parameters to Assess Few Scientific Impact Measures
}

\author{
P. VaraPrasada Rao \\ Associate Professor, \\ Department of CSE, \\ Gokaraju Rangaraju Institute of Engg\&Tech
}

\author{
A. Govardhan, PhD \\ Director, SIT \\ JNTUH, Hyderabad
}

\begin{abstract}
The growing use of bibliometrics among researchers has showcased the emergence of various evaluators of scientific research at the author front as well as journal publishers. Several indices has been put forward since the importance of scientific evaluators has gained prominence. Among any such indices, h-index, g-index etc., has been widely used in literature. The regression analysis presented here focus on the citation parameters such as total docs, citable docs, references per doc etc., reported in SCIMago database to evaluate the dependence of these parameters on various indices such as $\mathrm{h}$-index, a-index, $\mathrm{m}$-index, q2 index, r-index, a-r index and e-index respectively. The regression analysis was performed to delineate the dependence of various citation features on index values. From the analysis, it is understood that in all cases except a-index, an increase in SJR value is suggested which means that SJR contributes positively to enhance index factor of journals.
\end{abstract}

\section{Keywords}

SCIMago, bibliometrics, indexvalue, python

\section{INTRODUCTION}

Bibliometric indicators are intended to quantify the significance of individual journals and the scholarly publication in which research is published [1]. The increasing importance of the scientific impact of journals as independent measures of quality or impact of any scientific publication has triggered the evolution of many bibliometric indices [2] [3]. The impact of a scientific publication is measured by the number of times the article gets cited in other journals [4]. Bibliometric indicators quantify the pertinent citation and use of bibliographic information and are being used extensively in the assessment of research performance. Publication counts serve as an indicator of the amount of new scientific knowledge produced by researchers. The impact of this new knowledge can be measured by the number of times publications have been cited by other scientists in subsequent work. Evaluation of quality and quantity of publications can be done using a set of statistical and mathematical indices called bibliometric indicators [4] Indicators such as Quantitative indicators measure the productivity of the researcher and performance indicators measure the quality of the journal or researcher.

The validity of bibliometric indicators is much greater at research groups, university departments and research institutes and should be applied with extreme caution when measuring or comparing the performance of individual scientists.Bibliometric analysis of scientific activity is based on the assumption that carrying out research and communicating the results go hand in hand. Scientific progress is attained by researchers getting together to study specific research topics. Publications are regarded as the definitive statements of the results of research projects. This can be quantified and analysed to determine the size and nature of the research carried out. The need for a relatively quick and easy alternative to peer review for evaluating research performance led to the discovery of bibliometrics. [5].

The knowledge of research indices started when Hirsh proposed the h-index, designed to measure the impact of research publications to estimate the author influence [6]. $\mathrm{H}$-index has been regarded as the most reliable, robust and easily computed [7][8][9]. H-index assesses both the quantity and importance or relevance of publications [10]. $\mathrm{H}$-index has some limitations, and hence to overcome and provide enhancements to H-index, Egghe proposed the gindex [11]. Based on properties of $\mathrm{h}$ and $\mathrm{g}$ indices, Kosmulski [12] proposed the H(2)-index which concentrates on highly cited research publications. Other indices were proposed which concentrated on the publications that were located at (H-core) in its calculations [13] such as A-index proposed by Jin [14] where, the average number of citations for those publications in the $\mathrm{H}$-core is evaluated. As a variation of A-index, Bornmann et al proposed m-index where, instead of arithmetic average, median is employed as the measure of central tendency [14]. m-index calculated by dividing the $h$ index by the number of years of that journal's publication [15].In order to increase the index value of a journal or researcher, several policies can be adopted to improve citations, such as publishing more review articles, as well as inviting papers and request authors to cite work published. Therefore, here we present a methodology to assess the bibliometric index on a journal and important parameters that might influence a high index value.

\section{MATERIALS AND METHODS}

\subsection{SCIMago}

[16]Database includes journals from the information contained in Scopus database [17].SCImago database searched for 'computer science journals' and are listed. The journal parameters such Sci Journal Ranking (SJR), hindex, Total Docs, Total References, Total cites, citable docs, cites/doc and references/doc etc., are calculated by SCImago and are used.

\subsection{Regression}

Regression analysis is the method of correlating parameters with dependent variable. A python program was written to perform linear regression analysis. Bibliometrics indices were regarded as dependent variable 
and all other citation parameters are considered as independent variables. To obtain reliable and robust regression, it is desirable to consider a large dataset that covers reasonable diversity.

\subsection{Dataset}

SCImago site was searched for all categories of computer science journals and from the search result, nearly 142 journal data (Table 1) was selected as independent variables and various indices are presented as dependent variables. The relationship between dependent variable (bibliometric indices) and independent variables (citation parameters) was established by linear regression analysis. The generated equation was judged based on the parameters like correlation coefficient (r), $r^{2}$ and adjusted $r^{2}$.

\subsection{Bibliometric indices}

In recent years, several research and publications related indices were proposed to assess the quality of the academic research publications. Each one of those indices has its own strengths and weaknesses. Here, we considered indices such as h-index, a-index, m-index, $\mathrm{q} 2$ index, $\mathrm{r}$ index, a-r index and e-index respectively. The regression analysis was performed to delineate the dependence of various citation features on index values.

Table 1: Citation parameters from SCIMago and various bibliometric indices selected in analysis.

\begin{tabular}{|c|c|c|c|c|c|c|c|c|c|c|c|c|c|c|}
\hline Title & TD3 & TR3 & TC3 & CD3 & CD2 & RD & SJR & $\begin{array}{c}\mathbf{H} \\
\text { inde } \\
\mathbf{X}\end{array}$ & $\begin{array}{c}\mathbf{A} \\
\text { inde } \\
\mathbf{x}\end{array}$ & $\begin{array}{c}\mathbf{m} \\
\text { inde } \\
\mathbf{x}\end{array}$ & $\begin{array}{c}\mathbf{q 2} \\
\text { inde } \\
\mathbf{x}\end{array}$ & $\begin{array}{c}\mathbf{r} \\
\text { inde } \\
\mathbf{x}\end{array}$ & $\begin{array}{c}\text { a-r } \\
\text { inde } \\
x\end{array}$ & $\begin{array}{c}\mathrm{e} \\
\text { inde } \\
\mathbf{x}\end{array}$ \\
\hline $\begin{array}{c}\text { Archives of } \\
\text { Computational } \\
\text { Methods in } \\
\text { Engineering }\end{array}$ & 42 & 1383 & 312 & 40 & 4.82 & $\begin{array}{c}98.7 \\
9\end{array}$ & $\begin{array}{c}6.28 \\
4\end{array}$ & 32 & 9.75 & $\begin{array}{c}10.6 \\
7\end{array}$ & $\begin{array}{c}18.4 \\
8\end{array}$ & $\begin{array}{c}99.9 \\
2\end{array}$ & $\begin{array}{c}57.6 \\
9\end{array}$ & $\begin{array}{c}16.7 \\
3\end{array}$ \\
\hline $\begin{array}{l}\text { MIS Quarterly: } \\
\text { Management } \\
\text { Information }\end{array}$ & 151 & 5571 & 1771 & 149 & 9.88 & $\begin{array}{c}81.9 \\
3\end{array}$ & $\begin{array}{c}6.25 \\
1\end{array}$ & 132 & $\begin{array}{c}13.4 \\
2\end{array}$ & 44 & $\begin{array}{c}76.2 \\
1\end{array}$ & $\begin{array}{c}483 . \\
5\end{array}$ & $\begin{array}{c}279 . \\
15\end{array}$ & $\begin{array}{c}40.4 \\
8\end{array}$ \\
\hline $\begin{array}{c}\text { Swarm and } \\
\text { Evolutionary } \\
\text { Computation }\end{array}$ & 46 & 1696 & 557 & 45 & $\begin{array}{c}12.3 \\
8\end{array}$ & $\begin{array}{c}41.3 \\
7\end{array}$ & $\begin{array}{c}5.63 \\
1\end{array}$ & 13 & $\begin{array}{c}42.8 \\
5\end{array}$ & 4.33 & 7.51 & $\begin{array}{c}85.0 \\
9\end{array}$ & $\begin{array}{c}49.1 \\
3\end{array}$ & $\begin{array}{c}23.3 \\
2\end{array}$ \\
\hline $\begin{array}{c}\text { Proceedings of the } \\
\text { Annual ACM } \\
\text { Symposium on } \\
\text { Theory of } \\
\text { Computing } \\
\end{array}$ & 260 & 3195 & 737 & 253 & 2.57 & $\begin{array}{c}31.6 \\
3\end{array}$ & $\begin{array}{c}4.47 \\
9\end{array}$ & 40 & $\begin{array}{c}18.4 \\
3\end{array}$ & $\begin{array}{c}13.3 \\
3\end{array}$ & $\begin{array}{c}23.0 \\
9\end{array}$ & $\begin{array}{c}171 . \\
7\end{array}$ & $\begin{array}{c}99.1 \\
3\end{array}$ & 26.4 \\
\hline $\begin{array}{l}\text { IEEE Wireless } \\
\text { Communications }\end{array}$ & 238 & 1368 & 1693 & 211 & 7.16 & $\begin{array}{c}12.1 \\
1\end{array}$ & 3.83 & 98 & $\begin{array}{c}17.2 \\
8\end{array}$ & $\begin{array}{c}32.6 \\
7\end{array}$ & $\begin{array}{c}56.5 \\
8\end{array}$ & $\begin{array}{c}407 . \\
33\end{array}$ & $\begin{array}{c}235 . \\
17\end{array}$ & $\begin{array}{c}39.9 \\
4\end{array}$ \\
\hline $\begin{array}{c}\text { IEEE Transactions } \\
\text { on Information } \\
\text { Theory } \\
\end{array}$ & 1565 & $\begin{array}{c}1700 \\
6\end{array}$ & 6546 & 1544 & 3.77 & $\begin{array}{c}30.3 \\
1\end{array}$ & $\begin{array}{c}3.39 \\
7\end{array}$ & 192 & $\begin{array}{c}34.0 \\
9\end{array}$ & 64 & $\begin{array}{c}110 . \\
85\end{array}$ & $\begin{array}{c}1121 \\
.09\end{array}$ & $\begin{array}{c}647 . \\
26\end{array}$ & $\begin{array}{c}79.7 \\
1\end{array}$ \\
\hline $\begin{array}{c}\text { Journal of the } \\
\text { ACM }\end{array}$ & 92 & 1967 & 396 & 81 & 5.18 & $\begin{array}{c}43.7 \\
1\end{array}$ & $\begin{array}{c}3.35 \\
1\end{array}$ & 88 & 4.5 & $\begin{array}{c}29.3 \\
3\end{array}$ & $\begin{array}{c}50.8 \\
1\end{array}$ & $\begin{array}{c}186 . \\
68\end{array}$ & $\begin{array}{c}107 . \\
78\end{array}$ & $\begin{array}{c}17.5 \\
5\end{array}$ \\
\hline $\begin{array}{l}\text { IEEE Journal on } \\
\text { Selected Areas in } \\
\text { Communications }\end{array}$ & 534 & 8569 & 3107 & 502 & 6 & $\begin{array}{c}29.1 \\
5\end{array}$ & $\begin{array}{c}3.33 \\
5\end{array}$ & 165 & $\begin{array}{c}18.8 \\
3\end{array}$ & 55 & $\begin{array}{c}95.2 \\
6\end{array}$ & 716 & $\begin{array}{c}413 . \\
38\end{array}$ & $\begin{array}{c}54.2 \\
4\end{array}$ \\
\hline $\begin{array}{c}\text { IEEE } \\
\text { Communications } \\
\text { Magazine }\end{array}$ & 829 & 2874 & 4817 & 643 & 7.3 & 9.94 & $\begin{array}{c}3.19 \\
6\end{array}$ & 144 & $\begin{array}{c}33.4 \\
5\end{array}$ & 48 & $\begin{array}{c}83.1 \\
4\end{array}$ & $\begin{array}{c}832 . \\
86\end{array}$ & $\begin{array}{c}480 . \\
85\end{array}$ & $\begin{array}{c}68.3 \\
6\end{array}$ \\
\hline $\begin{array}{l}\text { Journal of Strategic } \\
\text { Information } \\
\text { Systems }\end{array}$ & 85 & 1715 & 334 & 72 & 4.39 & $\begin{array}{c}57.1 \\
7\end{array}$ & $\begin{array}{c}2.90 \\
3\end{array}$ & 50 & 6.68 & $\begin{array}{c}16.6 \\
7\end{array}$ & $\begin{array}{c}28.8 \\
7\end{array}$ & $\begin{array}{c}129 . \\
23\end{array}$ & $\begin{array}{c}74.6 \\
1\end{array}$ & $\begin{array}{c}16.8 \\
5\end{array}$ \\
\hline $\begin{array}{c}\text { IEEE Transactions } \\
\text { on Wireless } \\
\text { Communications } \\
\end{array}$ & 1354 & $\begin{array}{c}1643 \\
7\end{array}$ & 6025 & 1324 & 4.09 & $\begin{array}{c}27.5 \\
3\end{array}$ & $\begin{array}{c}2.72 \\
2\end{array}$ & 118 & $\begin{array}{c}51.0 \\
6\end{array}$ & $\begin{array}{c}39.3 \\
3\end{array}$ & $\begin{array}{c}68.1 \\
3\end{array}$ & $\begin{array}{c}843 . \\
18\end{array}$ & $\begin{array}{c}486 . \\
81\end{array}$ & $\begin{array}{c}76.8 \\
6\end{array}$ \\
\hline $\begin{array}{l}\text { Foundations and } \\
\text { Trends in } \\
\text { Information }\end{array}$ & 10 & 574 & 93 & 10 & 5.83 & $\begin{array}{c}191 . \\
33\end{array}$ & $\begin{array}{c}2.71 \\
4\end{array}$ & 15 & 6.2 & 5 & 8.66 & $\begin{array}{c}37.3 \\
5\end{array}$ & $\begin{array}{c}21.5 \\
6\end{array}$ & 8.83 \\
\hline $\begin{array}{c}\text { IEEE Transactions } \\
\text { on Industrial } \\
\text { Informatics } \\
\end{array}$ & 243 & 8691 & 2221 & 227 & $\begin{array}{c}11.1 \\
3\end{array}$ & $\begin{array}{c}35.9 \\
1\end{array}$ & $\begin{array}{c}2.66 \\
6\end{array}$ & 39 & $\begin{array}{c}56.9 \\
5\end{array}$ & 13 & $\begin{array}{c}22.5 \\
2\end{array}$ & $\begin{array}{c}294 . \\
31\end{array}$ & $\begin{array}{c}169 . \\
92\end{array}$ & $\begin{array}{c}46.7 \\
1\end{array}$ \\
\hline $\begin{array}{l}\text { Information } \\
\text { Sciences }\end{array}$ & 1147 & $\begin{array}{c}2573 \\
3\end{array}$ & 6125 & 1125 & 5.34 & $\begin{array}{c}39.7 \\
7\end{array}$ & $\begin{array}{c}2.60 \\
6\end{array}$ & 91 & $\begin{array}{c}67.3 \\
1\end{array}$ & $\begin{array}{c}30.3 \\
3\end{array}$ & $\begin{array}{c}52.5 \\
4\end{array}$ & $\begin{array}{c}746 . \\
58\end{array}$ & $\begin{array}{c}431 . \\
04\end{array}$ & $\begin{array}{c}77.6 \\
8\end{array}$ \\
\hline $\begin{array}{l}\text { Information and } \\
\text { Organization }\end{array}$ & 37 & 1267 & 138 & 36 & 4.23 & $\begin{array}{c}70.3 \\
9\end{array}$ & $\begin{array}{c}2.53 \\
4\end{array}$ & 34 & 4.06 & $\begin{array}{c}11.3 \\
3\end{array}$ & $\begin{array}{c}19.6 \\
3\end{array}$ & 68.5 & $\begin{array}{c}39.5 \\
5\end{array}$ & 10.2 \\
\hline Web Semantics & 132 & 902 & 437 & 104 & 4.61 & $\begin{array}{c}32.2 \\
1\end{array}$ & $\begin{array}{c}2.13 \\
1\end{array}$ & 49 & 8.92 & $\begin{array}{c}16.3 \\
3\end{array}$ & $\begin{array}{c}28.2 \\
9\end{array}$ & $\begin{array}{c}146 . \\
33\end{array}$ & $\begin{array}{c}84.4 \\
8\end{array}$ & 19.7 \\
\hline
\end{tabular}




\begin{tabular}{|c|c|c|c|c|c|c|c|c|c|c|c|c|c|c|}
\hline $\begin{array}{l}\text { IEEE/ACM } \\
\text { Transactions on } \\
\text { Networking }\end{array}$ & 446 & 4733 & 1691 & 415 & 3.63 & $\begin{array}{c}17.9 \\
3\end{array}$ & $\begin{array}{c}2.04 \\
2\end{array}$ & 124 & $\begin{array}{c}13.6 \\
4\end{array}$ & $\begin{array}{c}41.3 \\
3\end{array}$ & $\begin{array}{c}71.5 \\
9\end{array}$ & $\begin{array}{c}457 . \\
91\end{array}$ & $\begin{array}{c}264 . \\
38\end{array}$ & $\begin{array}{c}39.5 \\
9\end{array}$ \\
\hline $\begin{array}{l}\text { Enterprise } \\
\text { Information } \\
\text { Systems }\end{array}$ & 69 & 2296 & 351 & 65 & 6 & $\begin{array}{c}45.0 \\
2\end{array}$ & $\begin{array}{c}1.99 \\
4\end{array}$ & 21 & $\begin{array}{c}16.7 \\
1\end{array}$ & 7 & $\begin{array}{c}12.1 \\
2\end{array}$ & $\begin{array}{c}85.8 \\
5\end{array}$ & $\begin{array}{c}49.5 \\
7\end{array}$ & $\begin{array}{c}18.1 \\
7\end{array}$ \\
\hline $\begin{array}{c}\text { Journal of } \\
\text { Management } \\
\text { Information }\end{array}$ & 138 & 2513 & 445 & 122 & 2.94 & $\begin{array}{c}57.1 \\
1\end{array}$ & $\begin{array}{c}1.96 \\
9\end{array}$ & 90 & 4.94 & 30 & $\begin{array}{c}51.9 \\
6\end{array}$ & $\begin{array}{c}200 . \\
12\end{array}$ & $\begin{array}{c}115 . \\
54\end{array}$ & $\begin{array}{c}18.8 \\
4\end{array}$ \\
\hline $\begin{array}{c}\text { Journal of } \\
\text { Computer- } \\
\text { Mediated } \\
\end{array}$ & 86 & 1416 & 262 & 81 & 3.12 & $\begin{array}{c}50.5 \\
7\end{array}$ & $\begin{array}{c}1.95 \\
8\end{array}$ & 64 & 4.09 & $\begin{array}{c}21.3 \\
3\end{array}$ & $\begin{array}{c}36.9 \\
5\end{array}$ & $\begin{array}{c}129 . \\
49\end{array}$ & $\begin{array}{c}74.7 \\
6\end{array}$ & $\begin{array}{c}14.0 \\
7\end{array}$ \\
\hline $\begin{array}{l}\text { Communications } \\
\text { of the ACM }\end{array}$ & 1017 & 2013 & 2955 & 650 & 3.57 & 7.51 & $\begin{array}{c}1.82 \\
2\end{array}$ & 131 & $\begin{array}{c}22.5 \\
6\end{array}$ & $\begin{array}{c}43.6 \\
7\end{array}$ & $\begin{array}{c}75.6 \\
3\end{array}$ & $\begin{array}{c}622 . \\
18\end{array}$ & $\begin{array}{c}359 . \\
21\end{array}$ & $\begin{array}{c}53.1 \\
4\end{array}$ \\
\hline $\begin{array}{l}\text { Decision Support } \\
\text { Systems }\end{array}$ & 481 & 9942 & 1590 & 469 & 3.14 & 43.8 & $\begin{array}{c}1.81 \\
4\end{array}$ & 76 & $\begin{array}{c}20.9 \\
2\end{array}$ & $\begin{array}{c}25.3 \\
3\end{array}$ & $\begin{array}{c}43.8 \\
8\end{array}$ & $\begin{array}{c}347 . \\
62\end{array}$ & $\begin{array}{c}200 . \\
7\end{array}$ & $\begin{array}{c}38.9 \\
1\end{array}$ \\
\hline $\begin{array}{c}\text { Information } \\
\text { Systems Journal }\end{array}$ & 82 & 1817 & 229 & 63 & 2.43 & $\begin{array}{c}50.4 \\
7\end{array}$ & $\begin{array}{c}1.79 \\
8\end{array}$ & 52 & 4.4 & $\begin{array}{c}17.3 \\
3\end{array}$ & $\begin{array}{c}30.0 \\
2\end{array}$ & $\begin{array}{c}109 . \\
12\end{array}$ & 63 & 13.3 \\
\hline IEEE Network & 152 & 806 & 775 & 119 & 6.07 & $\begin{array}{c}12.0 \\
3\end{array}$ & $\begin{array}{c}1.79 \\
6\end{array}$ & 80 & 9.69 & $\begin{array}{c}26.6 \\
7\end{array}$ & $\begin{array}{c}46.1 \\
9\end{array}$ & 249 & $\begin{array}{c}143 . \\
76\end{array}$ & $\begin{array}{c}26.3 \\
6\end{array}$ \\
\hline $\begin{array}{l}\text { Journal of the } \\
\text { American Society } \\
\text { for Information }\end{array}$ & 602 & $\begin{array}{c}1105 \\
5\end{array}$ & 1867 & 534 & 3.3 & $\begin{array}{c}43.8 \\
7\end{array}$ & $\begin{array}{c}1.74 \\
5\end{array}$ & 83 & $\begin{array}{c}22.4 \\
9\end{array}$ & $\begin{array}{c}27.6 \\
7\end{array}$ & $\begin{array}{c}47.9 \\
2\end{array}$ & $\begin{array}{c}393 . \\
65\end{array}$ & $\begin{array}{c}227 . \\
27\end{array}$ & $\begin{array}{c}42.2 \\
4\end{array}$ \\
\hline $\begin{array}{c}\text { Journal of } \\
\text { Information } \\
\text { Technology }\end{array}$ & 110 & 1760 & 264 & 67 & 2.49 & $\begin{array}{c}73.3 \\
3\end{array}$ & $\begin{array}{c}1.65 \\
9\end{array}$ & 43 & 6.14 & $\begin{array}{c}14.3 \\
3\end{array}$ & $\begin{array}{c}24.8 \\
3\end{array}$ & $\begin{array}{c}106 . \\
55\end{array}$ & $\begin{array}{c}61.5 \\
1\end{array}$ & $\begin{array}{c}14.8 \\
7\end{array}$ \\
\hline $\begin{array}{l}\text { Journal of the } \\
\text { Association of } \\
\text { Information }\end{array}$ & 103 & 2386 & 331 & 103 & 2.46 & $\begin{array}{c}95.4 \\
4\end{array}$ & $\begin{array}{c}1.62 \\
5\end{array}$ & 31 & $\begin{array}{c}10.6 \\
8\end{array}$ & $\begin{array}{c}10.3 \\
3\end{array}$ & 17.9 & $\begin{array}{c}101 . \\
3\end{array}$ & $\begin{array}{c}58.4 \\
8\end{array}$ & $\begin{array}{c}17.3 \\
2\end{array}$ \\
\hline $\begin{array}{c}\text { Knowledge and } \\
\text { Information } \\
\text { Systems } \\
\end{array}$ & 294 & 8431 & 902 & 279 & 3.02 & $\begin{array}{c}46.3 \\
2\end{array}$ & $\begin{array}{c}1.61 \\
6\end{array}$ & 31 & 29.1 & $\begin{array}{c}10.3 \\
3\end{array}$ & 17.9 & $\begin{array}{c}167 . \\
22\end{array}$ & $\begin{array}{c}96.5 \\
4\end{array}$ & $\begin{array}{c}29.5 \\
1\end{array}$ \\
\hline $\begin{array}{l}\text { ACM Transactions } \\
\text { on the Web }\end{array}$ & 56 & 1551 & 255 & 54 & 4.68 & 51.7 & $\begin{array}{c}1.56 \\
3\end{array}$ & 26 & 9.81 & 8.67 & $\begin{array}{c}15.0 \\
1\end{array}$ & $\begin{array}{c}81.4 \\
2\end{array}$ & $\begin{array}{c}47.0 \\
1\end{array}$ & $\begin{array}{c}15.1 \\
3\end{array}$ \\
\hline $\begin{array}{l}\text { Information } \\
\text { Systems }\end{array}$ & 180 & 2950 & 596 & 158 & 3.4 & $\begin{array}{l}33.5 \\
2\end{array}$ & $\begin{array}{c}1.52 \\
9\end{array}$ & 53 & $\begin{array}{c}11.2 \\
5\end{array}$ & $\begin{array}{c}17.6 \\
7\end{array}$ & 30.6 & $\begin{array}{c}177 . \\
73\end{array}$ & $\begin{array}{c}102 . \\
61\end{array}$ & 23.3 \\
\hline $\begin{array}{c}\text { European Journal } \\
\text { of Information } \\
\text { Systems }\end{array}$ & 150 & 3347 & 428 & 143 & 2.76 & $\begin{array}{c}77.8 \\
4\end{array}$ & $\begin{array}{c}1.51 \\
5\end{array}$ & 58 & 7.38 & $\begin{array}{c}19.3 \\
3\end{array}$ & $\begin{array}{c}33.4 \\
9\end{array}$ & $\begin{array}{c}157 . \\
56\end{array}$ & $\begin{array}{c}90.9 \\
7\end{array}$ & $\begin{array}{c}19.2 \\
4\end{array}$ \\
\hline $\begin{array}{l}\text { ACM Transactions } \\
\text { on Programming } \\
\text { Languages and } \\
\end{array}$ & 59 & 799 & 148 & 54 & 2.86 & $\begin{array}{c}57.0 \\
7\end{array}$ & $\begin{array}{c}1.49 \\
2\end{array}$ & 51 & 2.9 & 17 & $\begin{array}{c}29.4 \\
4\end{array}$ & $\begin{array}{c}86.8 \\
8\end{array}$ & $\begin{array}{c}50.1 \\
6\end{array}$ & 9.85 \\
\hline $\begin{array}{c}\text { IEEE } \\
\text { Communications } \\
\text { Letters }\end{array}$ & 1378 & 6866 & 3030 & 1370 & 2.18 & $\begin{array}{c}11.2 \\
7\end{array}$ & 1.19 & 96 & $\begin{array}{c}31.5 \\
6\end{array}$ & 32 & $\begin{array}{c}55.4 \\
3\end{array}$ & $\begin{array}{c}539 . \\
33\end{array}$ & $\begin{array}{c}311 . \\
38\end{array}$ & $\begin{array}{c}54.1 \\
7\end{array}$ \\
\hline $\begin{array}{l}\text { IEEE Transactions } \\
\text { on Services } \\
\text { Computing }\end{array}$ & 104 & 1577 & 491 & 90 & 4.36 & $\begin{array}{c}34.2 \\
8\end{array}$ & $\begin{array}{c}1.17 \\
6\end{array}$ & 27 & $\begin{array}{c}18.1 \\
9\end{array}$ & 9 & $\begin{array}{c}15.5 \\
9\end{array}$ & $\begin{array}{c}115 . \\
14\end{array}$ & $\begin{array}{c}66.4 \\
8\end{array}$ & $\begin{array}{c}21.5 \\
4\end{array}$ \\
\hline $\begin{array}{l}\text { Journal of } \\
\text { Information } \\
\text { Systems }\end{array}$ & 46 & 1434 & 121 & 43 & 2.36 & $\begin{array}{c}55.1 \\
5\end{array}$ & $\begin{array}{c}1.15 \\
7\end{array}$ & 11 & 11 & 3.67 & 6.35 & $\begin{array}{c}36.4 \\
8\end{array}$ & $\begin{array}{c}21.0 \\
6\end{array}$ & $\begin{array}{c}10.4 \\
9\end{array}$ \\
\hline $\begin{array}{l}\text { ACM Transactions } \\
\text { on Management } \\
\text { Information } \\
\end{array}$ & 49 & 1352 & 109 & 48 & 2.31 & $\begin{array}{c}56.3 \\
3\end{array}$ & $\begin{array}{c}1.08 \\
5\end{array}$ & 7 & $\begin{array}{c}15.5 \\
7\end{array}$ & 2.33 & 4.04 & $\begin{array}{c}27.6 \\
2\end{array}$ & $\begin{array}{c}15.9 \\
5\end{array}$ & 10.1 \\
\hline $\begin{array}{c}\text { Information and } \\
\text { Software } \\
\text { Technology }\end{array}$ & 288 & 7370 & 915 & 267 & 3.07 & $\begin{array}{c}54.1 \\
9\end{array}$ & $\begin{array}{c}1.07 \\
2\end{array}$ & 54 & $\begin{array}{c}16.9 \\
4\end{array}$ & 18 & $\begin{array}{c}31.1 \\
8\end{array}$ & $\begin{array}{c}222 . \\
28\end{array}$ & $\begin{array}{c}128 . \\
34\end{array}$ & $\begin{array}{c}29.3 \\
4\end{array}$ \\
\hline $\begin{array}{c}\text { Software and } \\
\text { Systems Modelling }\end{array}$ & 128 & 5182 & 234 & 83 & 2.2 & $\begin{array}{c}40.4 \\
8\end{array}$ & $\begin{array}{c}1.05 \\
5\end{array}$ & 28 & 8.36 & 9.33 & $\begin{array}{c}16.1 \\
7\end{array}$ & $\begin{array}{c}80.9 \\
4\end{array}$ & $\begin{array}{c}46.7 \\
3\end{array}$ & $\begin{array}{c}14.3 \\
5\end{array}$ \\
\hline $\begin{array}{l}\text { IEEE Internet } \\
\text { Computing }\end{array}$ & 298 & 759 & 809 & 278 & 2.62 & 8.34 & $\begin{array}{c}1.04 \\
4\end{array}$ & 79 & $\begin{array}{c}10.2 \\
4\end{array}$ & $\begin{array}{c}26.3 \\
3\end{array}$ & $\begin{array}{c}45.6 \\
1\end{array}$ & $\begin{array}{c}252 . \\
81\end{array}$ & $\begin{array}{c}145 . \\
96\end{array}$ & $\begin{array}{c}27.0 \\
2\end{array}$ \\
\hline $\begin{array}{c}\text { Journal of } \\
\text { Computational } \\
\text { Science }\end{array}$ & 135 & 1361 & 320 & 123 & 2.6 & 16.6 & $\begin{array}{c}1.03 \\
8\end{array}$ & 11 & $\begin{array}{c}29.0 \\
9\end{array}$ & 3.67 & 6.35 & $\begin{array}{c}59.3 \\
3\end{array}$ & $\begin{array}{c}34.2 \\
5\end{array}$ & $\begin{array}{c}17.5 \\
8\end{array}$ \\
\hline
\end{tabular}




\begin{tabular}{|c|c|c|c|c|c|c|c|c|c|c|c|c|c|c|}
\hline $\begin{array}{l}\text { Information and } \\
\text { Computation }\end{array}$ & 230 & 3216 & 275 & 220 & 1.2 & $\begin{array}{c}20.3 \\
5\end{array}$ & $\begin{array}{c}1.02 \\
9\end{array}$ & 50 & 5.5 & $\begin{array}{c}16.6 \\
7\end{array}$ & $\begin{array}{c}28.8 \\
7\end{array}$ & $\begin{array}{c}117 . \\
26\end{array}$ & 67.7 & 15 \\
\hline Soft Computing & 485 & 9584 & 951 & 462 & 2.02 & 36.3 & $\begin{array}{c}1.01 \\
9\end{array}$ & 36 & $\begin{array}{c}26.4 \\
2\end{array}$ & 12 & $\begin{array}{c}20.7 \\
8\end{array}$ & $\begin{array}{c}185 . \\
03\end{array}$ & $\begin{array}{c}106 . \\
83\end{array}$ & $\begin{array}{c}30.2 \\
5\end{array}$ \\
\hline $\begin{array}{l}\text { Interacting with } \\
\text { Computers }\end{array}$ & 154 & 1683 & 421 & 147 & 2.03 & $\begin{array}{c}36.5 \\
9\end{array}$ & $\begin{array}{c}0.98 \\
9\end{array}$ & 47 & 8.96 & $\begin{array}{c}15.6 \\
7\end{array}$ & $\begin{array}{c}27.1 \\
4\end{array}$ & $\begin{array}{c}140 . \\
67\end{array}$ & $\begin{array}{c}81.2 \\
1\end{array}$ & $\begin{array}{c}19.3 \\
4\end{array}$ \\
\hline $\begin{array}{c}\text { International } \\
\text { Journal of Human } \\
\text { Computer Studies }\end{array}$ & 192 & 3961 & 505 & 186 & 1.94 & $\begin{array}{c}50.1 \\
4\end{array}$ & $\begin{array}{c}0.98 \\
8\end{array}$ & 76 & 6.64 & $\begin{array}{c}25.3 \\
3\end{array}$ & $\begin{array}{c}43.8 \\
8\end{array}$ & $\begin{array}{c}195 . \\
91\end{array}$ & $\begin{array}{c}113 . \\
11\end{array}$ & $\begin{array}{c}20.7 \\
1\end{array}$ \\
\hline $\begin{array}{c}\text { Journal of } \\
\text { Computer Security }\end{array}$ & 98 & 1220 & 183 & 84 & 2 & $\begin{array}{c}42.0 \\
7\end{array}$ & $\begin{array}{c}0.97 \\
1\end{array}$ & 40 & 4.58 & $\begin{array}{c}13.3 \\
3\end{array}$ & $\begin{array}{c}23.0 \\
9\end{array}$ & $\begin{array}{c}85.5 \\
6\end{array}$ & 49.4 & $\begin{array}{c}11.9 \\
6\end{array}$ \\
\hline $\begin{array}{c}\text { Journal of } \\
\text { Educational } \\
\text { Computing }\end{array}$ & 128 & 1747 & 166 & 128 & 0.99 & $\begin{array}{c}43.6 \\
8\end{array}$ & 0.84 & 35 & 4.74 & $\begin{array}{c}11.6 \\
7\end{array}$ & $\begin{array}{c}20.2 \\
1\end{array}$ & $\begin{array}{c}76.2 \\
2\end{array}$ & $\begin{array}{c}44.0 \\
1\end{array}$ & $\begin{array}{c}11.4 \\
5\end{array}$ \\
\hline $\begin{array}{l}\text { Computers and } \\
\text { Security }\end{array}$ & 210 & 3667 & 505 & 182 & 2.41 & 37.8 & $\begin{array}{c}0.83 \\
8\end{array}$ & 51 & 9.9 & 17 & $\begin{array}{c}29.4 \\
4\end{array}$ & $\begin{array}{c}160 . \\
48\end{array}$ & $\begin{array}{c}92.6 \\
6\end{array}$ & $\begin{array}{c}21.3 \\
1\end{array}$ \\
\hline $\begin{array}{c}\text { Information } \\
\text { Systems Research }\end{array}$ & 184 & 4437 & 853 & 174 & 3.99 & $\begin{array}{c}70.4 \\
3\end{array}$ & $\begin{array}{c}3.63 \\
2\end{array}$ & 99 & 8.62 & 33 & $\begin{array}{c}57.1 \\
6\end{array}$ & $\begin{array}{c}290 . \\
6\end{array}$ & $\begin{array}{c}167 . \\
78\end{array}$ & $\begin{array}{c}27.4 \\
6\end{array}$ \\
\hline $\begin{array}{c}\text { Journal of } \\
\text { Operations } \\
\text { Management } \\
\end{array}$ & 141 & 2808 & 916 & 130 & 5.32 & $\begin{array}{c}75.8 \\
9\end{array}$ & $\begin{array}{c}5.87 \\
2\end{array}$ & 108 & 8.48 & 36 & $\begin{array}{c}62.3 \\
5\end{array}$ & $\begin{array}{c}314 . \\
53\end{array}$ & $\begin{array}{c}181 . \\
59\end{array}$ & $\begin{array}{c}28.4 \\
3\end{array}$ \\
\hline $\begin{array}{l}\text { IEEE Transactions } \\
\text { on Fuzzy Systems }\end{array}$ & 285 & 4301 & 2487 & 281 & 8.42 & $\begin{array}{c}45.7 \\
6\end{array}$ & 3.59 & 119 & 20.9 & $\begin{array}{c}39.6 \\
7\end{array}$ & 68.7 & $\begin{array}{c}544 . \\
02\end{array}$ & $\begin{array}{c}314 . \\
09\end{array}$ & $\begin{array}{c}48.6 \\
6\end{array}$ \\
\hline $\begin{array}{l}\text { Mathematical } \\
\text { Programming } \\
\text { Computation }\end{array}$ & 36 & 495 & 157 & 36 & 4.12 & $\begin{array}{c}38.0 \\
8\end{array}$ & $\begin{array}{c}3.33 \\
5\end{array}$ & 11 & $\begin{array}{c}14.2 \\
7\end{array}$ & 3.67 & 6.35 & $\begin{array}{c}41.5 \\
6\end{array}$ & $\begin{array}{c}23.9 \\
9\end{array}$ & $\begin{array}{c}12.0 \\
8\end{array}$ \\
\hline $\begin{array}{c}\text { International } \\
\text { Journal of Robotics } \\
\text { Research } \\
\end{array}$ & 307 & 4035 & 1586 & 286 & 5.46 & $\begin{array}{c}44.3 \\
4\end{array}$ & 3.33 & 89 & $\begin{array}{c}17.8 \\
2\end{array}$ & $\begin{array}{c}29.6 \\
7\end{array}$ & $\begin{array}{c}51.3 \\
8\end{array}$ & $\begin{array}{c}375 . \\
7\end{array}$ & $\begin{array}{c}216 . \\
91\end{array}$ & $\begin{array}{c}38.6 \\
9\end{array}$ \\
\hline $\begin{array}{c}\text { IEEE Transactions } \\
\text { on Automatic } \\
\text { Control } \\
\end{array}$ & 1073 & 9995 & 5450 & 1056 & 4.58 & $\begin{array}{c}27.4 \\
6\end{array}$ & $\begin{array}{c}2.99 \\
2\end{array}$ & 175 & $\begin{array}{c}31.1 \\
4\end{array}$ & $\begin{array}{c}58.3 \\
3\end{array}$ & $\begin{array}{c}101 . \\
04\end{array}$ & $\begin{array}{c}976 . \\
6\end{array}$ & $\begin{array}{c}563 . \\
84\end{array}$ & $\begin{array}{c}72.6 \\
3\end{array}$ \\
\hline $\begin{array}{c}\text { Computers and } \\
\text { Operations } \\
\text { Research } \\
\end{array}$ & 721 & 9080 & 2518 & 698 & 3.03 & $\begin{array}{c}28.7 \\
3\end{array}$ & 2.97 & 84 & $\begin{array}{c}29.9 \\
8\end{array}$ & 28 & 48.5 & $\begin{array}{c}459 . \\
9\end{array}$ & $\begin{array}{c}265 . \\
53\end{array}$ & $\begin{array}{c}49.3 \\
4\end{array}$ \\
\hline $\begin{array}{c}\text { IEEE Transactions } \\
\text { on Signal } \\
\text { Processing } \\
\end{array}$ & 1709 & $\begin{array}{c}1670 \\
1\end{array}$ & 8058 & 1690 & 4.45 & $\begin{array}{c}34.1 \\
5\end{array}$ & $\begin{array}{c}2.81 \\
7\end{array}$ & 162 & $\begin{array}{c}49.7 \\
4\end{array}$ & 54 & $\begin{array}{c}93.5 \\
3\end{array}$ & $\begin{array}{c}1142 \\
.54\end{array}$ & $\begin{array}{c}659 . \\
65\end{array}$ & $\begin{array}{c}88.8 \\
6\end{array}$ \\
\hline $\begin{array}{c}\text { IEEE Journal on } \\
\text { Selected Topics in } \\
\text { Signal Processing } \\
\end{array}$ & 312 & 2508 & 1771 & 288 & 5.32 & $\begin{array}{c}30.9 \\
6\end{array}$ & $\begin{array}{c}2.70 \\
5\end{array}$ & 45 & $\begin{array}{c}39.3 \\
6\end{array}$ & 15 & $\begin{array}{c}25.9 \\
8\end{array}$ & $\begin{array}{c}282 . \\
3\end{array}$ & $\begin{array}{c}162 . \\
99\end{array}$ & $\begin{array}{c}41.5 \\
5\end{array}$ \\
\hline $\begin{array}{l}\text { IEEE Transactions } \\
\text { on Robotics }\end{array}$ & 330 & 4613 & 1845 & 329 & 5.03 & 37.5 & $\begin{array}{c}2.62 \\
6\end{array}$ & 77 & $\begin{array}{c}23.9 \\
6\end{array}$ & $\begin{array}{c}25.6 \\
7\end{array}$ & $\begin{array}{c}44.4 \\
6\end{array}$ & $\begin{array}{c}376 . \\
92\end{array}$ & $\begin{array}{c}217 . \\
61\end{array}$ & $\begin{array}{c}42.0 \\
5\end{array}$ \\
\hline $\begin{array}{c}\text { IEEE Transactions } \\
\text { on Image } \\
\text { Processing } \\
\end{array}$ & 973 & $\begin{array}{c}1587 \\
6\end{array}$ & 5752 & 965 & 5.01 & $\begin{array}{c}38.0 \\
7\end{array}$ & 1.98 & 169 & $\begin{array}{c}34.0 \\
4\end{array}$ & $\begin{array}{c}56.3 \\
3\end{array}$ & $\begin{array}{l}97.5 \\
7\end{array}$ & $\begin{array}{c}985 . \\
95\end{array}$ & $\begin{array}{c}569 . \\
24\end{array}$ & $\begin{array}{c}74.7 \\
2\end{array}$ \\
\hline $\begin{array}{l}\text { Medical Image } \\
\text { Analysis }\end{array}$ & 251 & 4984 & 1381 & 243 & 5.23 & $\begin{array}{c}50.3 \\
4\end{array}$ & $\begin{array}{c}1.97 \\
7\end{array}$ & 76 & $\begin{array}{c}18.1 \\
7\end{array}$ & $\begin{array}{c}25.3 \\
3\end{array}$ & $\begin{array}{c}43.8 \\
8\end{array}$ & $\begin{array}{c}323 . \\
97\end{array}$ & $\begin{array}{c}187 . \\
04\end{array}$ & $\begin{array}{c}36.1 \\
2\end{array}$ \\
\hline $\begin{array}{l}\text { Computers and } \\
\text { Structures }\end{array}$ & 497 & 7475 & 1481 & 483 & 2.84 & $\begin{array}{c}34.6 \\
1\end{array}$ & $\begin{array}{c}1.91 \\
9\end{array}$ & 75 & $\begin{array}{c}19.7 \\
5\end{array}$ & 25 & 43.3 & $\begin{array}{c}333 . \\
28\end{array}$ & $\begin{array}{c}192 . \\
42\end{array}$ & 37.5 \\
\hline $\begin{array}{l}\text { ACM Transactions } \\
\text { on Database } \\
\text { Systems }\end{array}$ & 88 & 1192 & 227 & 84 & 2.52 & $\begin{array}{c}42.5 \\
7\end{array}$ & $\begin{array}{c}1.83 \\
7\end{array}$ & 59 & 3.85 & $\begin{array}{c}19.6 \\
7\end{array}$ & $\begin{array}{c}34.0 \\
6\end{array}$ & $\begin{array}{c}115 . \\
73\end{array}$ & $\begin{array}{c}66.8 \\
2\end{array}$ & $\begin{array}{c}12.9 \\
6\end{array}$ \\
\hline $\begin{array}{l}\text { IEEE Signal } \\
\text { Processing } \\
\text { Magazine } \\
\end{array}$ & 334 & 3058 & 1507 & 280 & 5.26 & $\begin{array}{c}25.4 \\
8\end{array}$ & $\begin{array}{c}1.83 \\
1\end{array}$ & 106 & $\begin{array}{c}14.2 \\
2\end{array}$ & $\begin{array}{c}35.3 \\
3\end{array}$ & 61.2 & $\begin{array}{c}399 . \\
68\end{array}$ & $\begin{array}{c}230 . \\
75\end{array}$ & $\begin{array}{c}37.4 \\
3\end{array}$ \\
\hline $\begin{array}{c}\text { Journal of Field } \\
\text { Robotics }\end{array}$ & 146 & 1781 & 555 & 136 & 3.88 & $\begin{array}{c}37.8 \\
9\end{array}$ & $\begin{array}{c}1.76 \\
5\end{array}$ & 52 & $\begin{array}{c}10.6 \\
7\end{array}$ & $\begin{array}{c}17.3 \\
3\end{array}$ & $\begin{array}{c}30.0 \\
2\end{array}$ & $\begin{array}{c}169 . \\
88\end{array}$ & $\begin{array}{c}98.0 \\
8\end{array}$ & $\begin{array}{c}22.4 \\
3\end{array}$ \\
\hline $\begin{array}{l}\text { IEEE Transactions } \\
\text { on Knowledge and } \\
\text { Data Engineering }\end{array}$ & 423 & 8304 & 1858 & 408 & 3.84 & $\begin{array}{c}38.6 \\
2\end{array}$ & $\begin{array}{c}1.76 \\
3\end{array}$ & 103 & $\begin{array}{c}18.0 \\
4\end{array}$ & $\begin{array}{c}34.3 \\
3\end{array}$ & $\begin{array}{c}59.4 \\
7\end{array}$ & $\begin{array}{c}437 . \\
46\end{array}$ & $\begin{array}{c}252 . \\
57\end{array}$ & $\begin{array}{c}41.8 \\
9\end{array}$ \\
\hline
\end{tabular}




\begin{tabular}{|c|c|c|c|c|c|c|c|c|c|c|c|c|c|c|}
\hline $\begin{array}{c}\text { Mechanical } \\
\text { Systems and Signal } \\
\text { Processing }\end{array}$ & 640 & 9342 & 2363 & 618 & 3.74 & $\begin{array}{c}29.5 \\
6\end{array}$ & $\begin{array}{c}1.71 \\
3\end{array}$ & 79 & $\begin{array}{c}29.9 \\
1\end{array}$ & $\begin{array}{c}26.3 \\
3\end{array}$ & $\begin{array}{c}45.6 \\
1\end{array}$ & $\begin{array}{c}432 . \\
06\end{array}$ & $\begin{array}{c}249 . \\
45\end{array}$ & $\begin{array}{c}47.7 \\
9\end{array}$ \\
\hline $\begin{array}{l}\text { IEEE Transactions } \\
\text { on Software } \\
\text { Engineering } \\
\end{array}$ & 194 & 5253 & 996 & 180 & 5.29 & 53.6 & $\begin{array}{c}1.67 \\
4\end{array}$ & 111 & 8.97 & 37 & $\begin{array}{c}64.0 \\
9\end{array}$ & $\begin{array}{c}332 . \\
5\end{array}$ & $\begin{array}{c}191 . \\
97\end{array}$ & $\begin{array}{c}29.7 \\
5\end{array}$ \\
\hline $\begin{array}{c}\text { Pattern } \\
\text { Recognition }\end{array}$ & 964 & $\begin{array}{c}1166 \\
8\end{array}$ & 4210 & 956 & 4.03 & $\begin{array}{c}38.6 \\
4\end{array}$ & $\begin{array}{c}1.65 \\
3\end{array}$ & 121 & $\begin{array}{c}34.7 \\
9\end{array}$ & $\begin{array}{c}40.3 \\
3\end{array}$ & $\begin{array}{c}69.8 \\
6\end{array}$ & $\begin{array}{c}713 . \\
73\end{array}$ & $\begin{array}{c}412 . \\
07\end{array}$ & $\begin{array}{c}63.9 \\
5\end{array}$ \\
\hline Machine Learning & 174 & 4044 & 563 & 161 & 3.37 & $\begin{array}{c}43.9 \\
6\end{array}$ & $\begin{array}{c}1.65 \\
1\end{array}$ & 103 & 5.47 & $\begin{array}{c}34.3 \\
3\end{array}$ & $\begin{array}{c}59.4 \\
7\end{array}$ & $\begin{array}{c}240 . \\
81\end{array}$ & $\begin{array}{c}139 . \\
03\end{array}$ & $\begin{array}{c}21.4 \\
5\end{array}$ \\
\hline $\begin{array}{c}\text { Journal of } \\
\text { Computer and } \\
\text { System Sciences }\end{array}$ & 277 & 2556 & 538 & 245 & 2.21 & 28.4 & $\begin{array}{c}1.60 \\
5\end{array}$ & 56 & 9.61 & $\begin{array}{c}18.6 \\
7\end{array}$ & $\begin{array}{c}32.3 \\
3\end{array}$ & $\begin{array}{c}173 . \\
57\end{array}$ & $\begin{array}{c}100 . \\
21\end{array}$ & $\begin{array}{c}21.9 \\
5\end{array}$ \\
\hline $\begin{array}{l}\text { Fuzzy Sets and } \\
\text { Systems }\end{array}$ & 566 & 5404 & 1403 & 541 & 2.42 & $\begin{array}{c}23.0 \\
9\end{array}$ & $\begin{array}{c}1.55 \\
8\end{array}$ & 110 & $\begin{array}{c}12.7 \\
5\end{array}$ & $\begin{array}{c}36.6 \\
7\end{array}$ & $\begin{array}{c}63.5 \\
1\end{array}$ & $\begin{array}{c}392 . \\
85\end{array}$ & $\begin{array}{c}226 . \\
81\end{array}$ & $\begin{array}{c}35.9 \\
6\end{array}$ \\
\hline $\begin{array}{l}\text { Data Mining and } \\
\text { Knowledge } \\
\text { Discovery }\end{array}$ & 124 & 1717 & 414 & 113 & 3.54 & $\begin{array}{c}40.8 \\
8\end{array}$ & $\begin{array}{c}1.55 \\
8\end{array}$ & 62 & 6.68 & $\begin{array}{c}20.6 \\
7\end{array}$ & 35.8 & $\begin{array}{c}160 . \\
21\end{array}$ & 92.5 & $\begin{array}{c}18.7 \\
6\end{array}$ \\
\hline $\begin{array}{l}\text { ACM Transactions } \\
\text { on Information and } \\
\text { System Security }\end{array}$ & 72 & 730 & 233 & 66 & 3.08 & $\begin{array}{c}45.6 \\
3\end{array}$ & 1.55 & 41 & 5.68 & $\begin{array}{c}13.6 \\
7\end{array}$ & $\begin{array}{c}23.6 \\
7\end{array}$ & $\begin{array}{c}97.7 \\
4\end{array}$ & $\begin{array}{c}56.4 \\
3\end{array}$ & $\begin{array}{c}13.8 \\
6\end{array}$ \\
\hline $\begin{array}{c}\text { Computational } \\
\text { Statistics and Data } \\
\text { Analysis } \\
\end{array}$ & 938 & 7299 & 1245 & 872 & 1.25 & $\begin{array}{c}26.0 \\
7\end{array}$ & $\begin{array}{c}1.39 \\
9\end{array}$ & 57 & $\begin{array}{c}21.8 \\
4\end{array}$ & 19 & $\begin{array}{c}32.9 \\
1\end{array}$ & $\begin{array}{c}266 . \\
39\end{array}$ & $\begin{array}{c}153 . \\
8\end{array}$ & $\begin{array}{c}34.4 \\
7\end{array}$ \\
\hline Networks & 187 & 1487 & 223 & 176 & 1.03 & $\begin{array}{c}27.5 \\
4\end{array}$ & $\begin{array}{c}1.36 \\
4\end{array}$ & 38 & 5.87 & $\begin{array}{c}12.6 \\
7\end{array}$ & $\begin{array}{c}21.9 \\
4\end{array}$ & $\begin{array}{c}92.0 \\
5\end{array}$ & $\begin{array}{c}53.1 \\
5\end{array}$ & 13.6 \\
\hline $\begin{array}{c}\text { Mathematics of } \\
\text { Operations } \\
\text { Research } \\
\end{array}$ & 126 & 1166 & 195 & 126 & 1.05 & $\begin{array}{c}30.6 \\
8\end{array}$ & $\begin{array}{c}1.32 \\
9\end{array}$ & 50 & 3.9 & $\begin{array}{c}16.6 \\
7\end{array}$ & $\begin{array}{c}28.8 \\
7\end{array}$ & $\begin{array}{c}98.7 \\
4\end{array}$ & $\begin{array}{c}57.0 \\
1\end{array}$ & $\begin{array}{c}12.0 \\
4\end{array}$ \\
\hline $\begin{array}{l}\text { IEEE Transactions } \\
\text { on Neural } \\
\text { Networks and } \\
\text { Learning Systems }\end{array}$ & 179 & 6852 & 917 & 178 & 5.15 & $\begin{array}{c}30.5 \\
9\end{array}$ & $\begin{array}{c}1.30 \\
9\end{array}$ & 16 & $\begin{array}{c}57.3 \\
1\end{array}$ & 5.33 & 9.24 & $\begin{array}{c}121 . \\
13\end{array}$ & $\begin{array}{c}69.9 \\
3\end{array}$ & $\begin{array}{c}30.0 \\
2\end{array}$ \\
\hline $\begin{array}{c}\text { IEEE Robotics and } \\
\text { Automation } \\
\text { Magazine } \\
\end{array}$ & 237 & 980 & 559 & 160 & 3.85 & $\begin{array}{c}12.5 \\
6\end{array}$ & 1.09 & 51 & $\begin{array}{c}10.9 \\
6\end{array}$ & 17 & $\begin{array}{c}29.4 \\
4\end{array}$ & $\begin{array}{c}168 . \\
85\end{array}$ & $\begin{array}{c}97.4 \\
8\end{array}$ & $\begin{array}{c}22.5 \\
4\end{array}$ \\
\hline $\begin{array}{l}\text { Information and } \\
\text { Computation }\end{array}$ & 230 & 3216 & 275 & 220 & 1.2 & $\begin{array}{c}20.3 \\
5\end{array}$ & $\begin{array}{c}1.02 \\
9\end{array}$ & 50 & 5.5 & $\begin{array}{c}16.6 \\
7\end{array}$ & $\begin{array}{c}28.8 \\
7\end{array}$ & $\begin{array}{c}117 . \\
26\end{array}$ & 67.7 & 15 \\
\hline $\begin{array}{c}\text { Topics in } \\
\text { Cognitive Science }\end{array}$ & 176 & 2553 & 331 & 152 & 1.68 & $\begin{array}{c}41.8 \\
5\end{array}$ & $\begin{array}{c}1.02 \\
8\end{array}$ & 19 & $\begin{array}{c}17.4 \\
2\end{array}$ & 6.33 & $\begin{array}{c}10.9 \\
7\end{array}$ & 79.3 & $\begin{array}{c}45.7 \\
9\end{array}$ & $\begin{array}{c}17.6 \\
6\end{array}$ \\
\hline $\begin{array}{c}\text { ACM Transactions } \\
\text { on Knowledge } \\
\text { Discovery from } \\
\text { Data }\end{array}$ & 63 & 844 & 188 & 58 & 2.62 & 42.2 & $\begin{array}{c}1.02 \\
3\end{array}$ & 21 & 8.95 & 7 & $\begin{array}{c}12.1 \\
2\end{array}$ & $\begin{array}{c}62.8 \\
3\end{array}$ & $\begin{array}{c}36.2 \\
8\end{array}$ & $\begin{array}{c}12.9 \\
2\end{array}$ \\
\hline $\begin{array}{l}\text { Robotics and } \\
\text { Computer- } \\
\text { Integrated } \\
\text { Manufacturing } \\
\end{array}$ & 268 & 3218 & 788 & 263 & 3.16 & $\begin{array}{c}30.9 \\
4\end{array}$ & $\begin{array}{c}1.27 \\
2\end{array}$ & 51 & $\begin{array}{c}15.4 \\
5\end{array}$ & 17 & $\begin{array}{c}29.4 \\
4\end{array}$ & $\begin{array}{c}200 . \\
47\end{array}$ & $\begin{array}{c}115 . \\
74\end{array}$ & $\begin{array}{c}27.1 \\
5\end{array}$ \\
\hline Signal Processing & 899 & $\begin{array}{c}1102 \\
6\end{array}$ & 2717 & 881 & 3.02 & $\begin{array}{c}31.8 \\
7\end{array}$ & $\begin{array}{c}1.11 \\
1\end{array}$ & 77 & $\begin{array}{c}35.2 \\
9\end{array}$ & $\begin{array}{c}25.6 \\
7\end{array}$ & $\begin{array}{c}44.4 \\
6\end{array}$ & $\begin{array}{c}457 . \\
39\end{array}$ & $\begin{array}{c}264 . \\
08\end{array}$ & $\begin{array}{c}51.3 \\
8\end{array}$ \\
\hline $\begin{array}{l}\text { Computational } \\
\text { Geosciences }\end{array}$ & 175 & 2924 & 356 & 170 & 2.21 & $\begin{array}{c}37.4 \\
9\end{array}$ & $\begin{array}{c}1.09 \\
4\end{array}$ & 34 & $\begin{array}{c}10.4 \\
7\end{array}$ & $\begin{array}{c}11.3 \\
3\end{array}$ & $\begin{array}{c}19.6 \\
3\end{array}$ & $\begin{array}{c}110 . \\
02\end{array}$ & $\begin{array}{c}63.5 \\
2\end{array}$ & $\begin{array}{c}17.9 \\
4\end{array}$ \\
\hline $\begin{array}{c}\text { IEEE Robotics and } \\
\text { Automation } \\
\text { Magazine } \\
\end{array}$ & 237 & 980 & 559 & 160 & 3.85 & $\begin{array}{c}12.5 \\
6\end{array}$ & 1.09 & 51 & $\begin{array}{c}10.9 \\
6\end{array}$ & 17 & $\begin{array}{c}29.4 \\
4\end{array}$ & $\begin{array}{c}168 . \\
85\end{array}$ & $\begin{array}{c}97.4 \\
8\end{array}$ & $\begin{array}{c}22.5 \\
4\end{array}$ \\
\hline $\begin{array}{c}\text { Computational } \\
\text { Materials Science }\end{array}$ & 1521 & $\begin{array}{c}1944 \\
8\end{array}$ & 3193 & 1496 & 2.11 & $\begin{array}{c}35.7 \\
5\end{array}$ & $\begin{array}{c}1.08 \\
6\end{array}$ & 59 & $\begin{array}{c}54.1 \\
2\end{array}$ & $\begin{array}{c}19.6 \\
7\end{array}$ & $\begin{array}{c}34.0 \\
6\end{array}$ & $\begin{array}{c}434 . \\
04\end{array}$ & $\begin{array}{c}250 . \\
59\end{array}$ & $\begin{array}{c}55.9 \\
8\end{array}$ \\
\hline $\begin{array}{c}\text { Nano } \\
\text { Communication } \\
\text { Networks }\end{array}$ & 84 & 610 & 232 & 77 & 2.48 & $\begin{array}{c}29.0 \\
5\end{array}$ & $\begin{array}{c}1.08 \\
2\end{array}$ & 11 & $\begin{array}{c}21.0 \\
9\end{array}$ & 3.67 & 6.35 & $\begin{array}{c}50.5 \\
2\end{array}$ & $\begin{array}{c}29.1 \\
7\end{array}$ & $\begin{array}{c}14.8 \\
7\end{array}$ \\
\hline
\end{tabular}




\begin{tabular}{|c|c|c|c|c|c|c|c|c|c|c|c|c|c|c|}
\hline $\begin{array}{l}\text { ACM Transactions } \\
\text { on Knowledge } \\
\text { Discovery from } \\
\text { Data }\end{array}$ & 63 & 844 & 188 & 58 & 2.62 & 42.2 & $\begin{array}{c}1.02 \\
3\end{array}$ & 21 & 8.95 & 7 & $\begin{array}{c}12.1 \\
2\end{array}$ & $\begin{array}{c}62.8 \\
3\end{array}$ & $\begin{array}{c}36.2 \\
8\end{array}$ & $\begin{array}{c}12.9 \\
2\end{array}$ \\
\hline $\begin{array}{c}\text { IEEE Signal } \\
\text { Processing Letters }\end{array}$ & 585 & 4047 & 1573 & 577 & 2.46 & $\begin{array}{c}14.1 \\
5\end{array}$ & $\begin{array}{c}1.01 \\
6\end{array}$ & 82 & $\begin{array}{c}19.1 \\
8\end{array}$ & $\begin{array}{c}27.3 \\
3\end{array}$ & $\begin{array}{c}47.3 \\
4\end{array}$ & $\begin{array}{c}359 . \\
15\end{array}$ & $\begin{array}{c}207 . \\
35\end{array}$ & $\begin{array}{c}38.6 \\
1\end{array}$ \\
\hline $\begin{array}{c}\text { Pattern } \\
\text { Recognition } \\
\text { Letters } \\
\end{array}$ & 832 & 8082 & 2273 & 800 & 2.36 & $\begin{array}{c}27.0 \\
3\end{array}$ & $\begin{array}{c}0.99 \\
5\end{array}$ & 92 & $\begin{array}{c}24.7 \\
1\end{array}$ & $\begin{array}{c}30.6 \\
7\end{array}$ & $\begin{array}{c}53.1 \\
2\end{array}$ & $\begin{array}{c}457 . \\
29\end{array}$ & $\begin{array}{c}264 . \\
02\end{array}$ & 46.7 \\
\hline $\begin{array}{l}\text { Journal of Discrete } \\
\text { Algorithms }\end{array}$ & 177 & 1034 & 167 & 166 & 0.94 & $\begin{array}{c}18.4 \\
6\end{array}$ & 0.99 & 19 & 8.79 & 6.33 & $\begin{array}{c}10.9 \\
7\end{array}$ & $\begin{array}{c}56.3 \\
3\end{array}$ & $\begin{array}{c}32.5 \\
2\end{array}$ & $\begin{array}{c}12.1 \\
7\end{array}$ \\
\hline $\begin{array}{c}\text { Journal of } \\
\text { Information Hiding } \\
\text { and Multimedia }\end{array}$ & 88 & 539 & 223 & 88 & 2.77 & $\begin{array}{c}21.5 \\
6\end{array}$ & $\begin{array}{c}0.98 \\
4\end{array}$ & 14 & $\begin{array}{c}15.9 \\
3\end{array}$ & 4.67 & 8.08 & $\begin{array}{c}55.8 \\
7\end{array}$ & $\begin{array}{c}32.2 \\
6\end{array}$ & $\begin{array}{c}14.4 \\
6\end{array}$ \\
\hline $\begin{array}{c}\text { Robotics and } \\
\text { Autonomous } \\
\text { Systems } \\
\end{array}$ & 373 & 5754 & 1007 & 347 & 2.61 & $\begin{array}{c}33.4 \\
5\end{array}$ & $\begin{array}{c}0.95 \\
4\end{array}$ & 70 & $\begin{array}{c}14.3 \\
9\end{array}$ & $\begin{array}{c}23.3 \\
3\end{array}$ & $\begin{array}{c}40.4 \\
1\end{array}$ & $\begin{array}{c}265 . \\
5\end{array}$ & $\begin{array}{c}153 . \\
29\end{array}$ & $\begin{array}{c}30.6 \\
1\end{array}$ \\
\hline IEEE Software & 341 & 629 & 505 & 305 & 1.57 & 6.05 & $\begin{array}{c}0.84 \\
2\end{array}$ & 72 & 7.01 & 24 & $\begin{array}{c}41.5 \\
7\end{array}$ & $\begin{array}{c}190 . \\
68\end{array}$ & $\begin{array}{c}110 . \\
09\end{array}$ & $\begin{array}{c}20.8 \\
1\end{array}$ \\
\hline $\begin{array}{c}\text { International } \\
\text { Journal of Sensor } \\
\text { Networks } \\
\end{array}$ & 118 & 1254 & 394 & 117 & 3.24 & $\begin{array}{c}26.1 \\
3\end{array}$ & 0.81 & 20 & 19.7 & 6.67 & $\begin{array}{c}11.5 \\
5\end{array}$ & $\begin{array}{c}88.7 \\
7\end{array}$ & $\begin{array}{c}51.2 \\
5\end{array}$ & $\begin{array}{c}19.3 \\
4\end{array}$ \\
\hline $\begin{array}{c}\text { Foundations and } \\
\text { Trends in Machine } \\
\text { Learning } \\
\end{array}$ & 10 & 467 & 281 & 10 & 8.14 & $\begin{array}{c}155 . \\
67\end{array}$ & $\begin{array}{c}12.0 \\
76\end{array}$ & 11 & $\begin{array}{c}25.5 \\
5\end{array}$ & 3.67 & 6.35 & 55.6 & 32.1 & $\begin{array}{c}16.4 \\
3\end{array}$ \\
\hline $\begin{array}{l}\text { IEEE Transactions } \\
\text { on Pattern Analysis } \\
\text { and Machine }\end{array}$ & 584 & $\begin{array}{c}1032 \\
5\end{array}$ & 6273 & 556 & 9.69 & $\begin{array}{c}45.6 \\
9\end{array}$ & $\begin{array}{c}6.59 \\
4\end{array}$ & 221 & $\begin{array}{c}28.3 \\
8\end{array}$ & $\begin{array}{c}73.6 \\
7\end{array}$ & $\begin{array}{c}127 . \\
59\end{array}$ & $\begin{array}{c}1177 \\
.43\end{array}$ & $\begin{array}{c}679 . \\
79\end{array}$ & $\begin{array}{c}77.7 \\
9\end{array}$ \\
\hline $\begin{array}{l}\text { Computer Methods } \\
\text { in Applied } \\
\text { Mechanics and } \\
\text { Engineering }\end{array}$ & 764 & $\begin{array}{c}1113 \\
7\end{array}$ & 2614 & 754 & 2.93 & $\begin{array}{c}44.3 \\
7\end{array}$ & $\begin{array}{c}3.25 \\
2\end{array}$ & 120 & $\begin{array}{c}21.7 \\
8\end{array}$ & 40 & $\begin{array}{c}69.2 \\
8\end{array}$ & $\begin{array}{c}560 . \\
07\end{array}$ & $\begin{array}{c}323 . \\
36\end{array}$ & $\begin{array}{c}49.9 \\
4\end{array}$ \\
\hline $\begin{array}{l}\text { ACM Computing } \\
\text { Surveys }\end{array}$ & 84 & 8460 & 876 & 84 & 9.91 & $\begin{array}{c}119 . \\
15\end{array}$ & $\begin{array}{c}3.11 \\
3\end{array}$ & 90 & 9.73 & 30 & $\begin{array}{c}51.9 \\
6\end{array}$ & $\begin{array}{c}280 . \\
78\end{array}$ & $\begin{array}{c}162 . \\
11\end{array}$ & $\begin{array}{c}28.0 \\
4\end{array}$ \\
\hline $\begin{array}{c}\text { IEEE Transactions } \\
\text { on Evolutionary } \\
\text { Computation }\end{array}$ & 159 & 2709 & 1363 & 153 & 9.21 & 52.1 & $\begin{array}{c}3.10 \\
2\end{array}$ & 111 & $\begin{array}{c}12.2 \\
8\end{array}$ & 37 & $\begin{array}{c}64.0 \\
9\end{array}$ & $\begin{array}{c}388 . \\
96\end{array}$ & $\begin{array}{c}224 . \\
57\end{array}$ & $\begin{array}{c}35.3 \\
8\end{array}$ \\
\hline $\begin{array}{l}\text { SIAM Journal on } \\
\text { Computing }\end{array}$ & 227 & 3087 & 450 & 221 & 1.92 & $\begin{array}{c}32.4 \\
9\end{array}$ & $\begin{array}{c}2.64 \\
3\end{array}$ & 68 & 6.62 & $\begin{array}{c}22.6 \\
7\end{array}$ & $\begin{array}{c}39.2 \\
6\end{array}$ & $\begin{array}{c}174 . \\
93\end{array}$ & 101 & $\begin{array}{c}19.5 \\
4\end{array}$ \\
\hline $\begin{array}{l}\text { Computers and } \\
\text { Education }\end{array}$ & 757 & $\begin{array}{c}1448 \\
2\end{array}$ & 3724 & 744 & 4.52 & $\begin{array}{c}50.6 \\
4\end{array}$ & $\begin{array}{c}2.55 \\
8\end{array}$ & 77 & $\begin{array}{c}48.3 \\
6\end{array}$ & $\begin{array}{c}25.6 \\
7\end{array}$ & $\begin{array}{c}44.4 \\
6\end{array}$ & $\begin{array}{c}535 . \\
49\end{array}$ & $\begin{array}{c}309 . \\
16\end{array}$ & $\begin{array}{c}60.3 \\
9\end{array}$ \\
\hline $\begin{array}{l}\text { Mathematical } \\
\text { Programming }\end{array}$ & 285 & 5880 & 670 & 272 & 2.43 & 30 & $\begin{array}{c}2.54 \\
2\end{array}$ & 75 & 8.93 & 25 & 43.3 & $\begin{array}{c}224 . \\
17\end{array}$ & $\begin{array}{c}129 . \\
42\end{array}$ & $\begin{array}{c}24.3 \\
9\end{array}$ \\
\hline $\begin{array}{c}\text { ACM Transactions } \\
\text { on Mathematical } \\
\text { Software } \\
\end{array}$ & 83 & 976 & 282 & 82 & 4 & $\begin{array}{c}32.5 \\
3\end{array}$ & $\begin{array}{c}2.32 \\
3\end{array}$ & 54 & 5.22 & 18 & $\begin{array}{c}31.1 \\
8\end{array}$ & $\begin{array}{c}123 . \\
4\end{array}$ & $\begin{array}{c}71.2 \\
5\end{array}$ & 15.1 \\
\hline $\begin{array}{c}\text { IEEE Transactions } \\
\text { on Mobile } \\
\text { Computing } \\
\end{array}$ & 414 & 6393 & 2034 & 401 & 4.75 & $\begin{array}{c}33.1 \\
2\end{array}$ & $\begin{array}{c}2.25 \\
8\end{array}$ & 80 & $\begin{array}{c}25.4 \\
3\end{array}$ & $\begin{array}{c}26.6 \\
7\end{array}$ & $\begin{array}{c}46.1 \\
9\end{array}$ & $\begin{array}{c}403 . \\
39\end{array}$ & $\begin{array}{c}232 . \\
89\end{array}$ & 44.2 \\
\hline $\begin{array}{l}\text { Computers and } \\
\text { Geotechnics }\end{array}$ & 329 & 4736 & 812 & 316 & 2.5 & $\begin{array}{c}33.3 \\
5\end{array}$ & $\begin{array}{c}2.24 \\
4\end{array}$ & 48 & $\begin{array}{c}16.9 \\
2\end{array}$ & 16 & $\begin{array}{c}27.7 \\
1\end{array}$ & $\begin{array}{c}197 . \\
42\end{array}$ & $\begin{array}{c}113 . \\
98\end{array}$ & $\begin{array}{c}27.6 \\
4\end{array}$ \\
\hline $\begin{array}{l}\text { Journal of Machine } \\
\text { Learning Research }\end{array}$ & 756 & 5817 & 2379 & 739 & 2.51 & $\begin{array}{c}33.8 \\
2\end{array}$ & $\begin{array}{c}2.18 \\
6\end{array}$ & 94 & $\begin{array}{c}25.3 \\
1\end{array}$ & $\begin{array}{c}31.3 \\
3\end{array}$ & $\begin{array}{c}54.2 \\
7\end{array}$ & $\begin{array}{c}472 . \\
89\end{array}$ & $\begin{array}{c}273 . \\
02\end{array}$ & 47.8 \\
\hline $\begin{array}{c}\text { Foundations of } \\
\text { Computational } \\
\text { Mathematics } \\
\end{array}$ & 78 & 1286 & 204 & 73 & 2.45 & $\begin{array}{c}29.9 \\
1\end{array}$ & $\begin{array}{c}2.18 \\
3\end{array}$ & 29 & 7.03 & 9.67 & $\begin{array}{c}16.7 \\
4\end{array}$ & $\begin{array}{c}76.9 \\
2\end{array}$ & $\begin{array}{c}44.4 \\
1\end{array}$ & $\begin{array}{c}13.2 \\
3\end{array}$ \\
\hline $\begin{array}{l}\text { Artificial } \\
\text { Intelligence }\end{array}$ & 203 & 3603 & 799 & 196 & 3.91 & $\begin{array}{c}51.4 \\
7\end{array}$ & $\begin{array}{c}2.17 \\
4\end{array}$ & 101 & 7.91 & $\begin{array}{c}33.6 \\
7\end{array}$ & $\begin{array}{c}58.3 \\
1\end{array}$ & $\begin{array}{c}284 . \\
08\end{array}$ & $\begin{array}{c}164 . \\
01\end{array}$ & $\begin{array}{c}26.4 \\
2\end{array}$ \\
\hline $\begin{array}{l}\text { Computational } \\
\text { Intelligence and } \\
\text { Neuroscience }\end{array}$ & 110 & 954 & 419 & 103 & 5.16 & $\begin{array}{c}50.2 \\
1\end{array}$ & 2.17 & 23 & $\begin{array}{c}18.2 \\
2\end{array}$ & 7.67 & $\begin{array}{c}13.2 \\
8\end{array}$ & $\begin{array}{c}98.1 \\
7\end{array}$ & $\begin{array}{c}56.6 \\
8\end{array}$ & 19.9 \\
\hline
\end{tabular}




\begin{tabular}{|c|c|c|c|c|c|c|c|c|c|c|c|c|c|c|}
\hline $\begin{array}{c}\text { Journal of } \\
\text { Computer Assisted } \\
\text { Learning }\end{array}$ & 138 & 2404 & 485 & 129 & 2.52 & $\begin{array}{c}46.2 \\
3\end{array}$ & $\begin{array}{c}2.14 \\
4\end{array}$ & 48 & 10.1 & 16 & $\begin{array}{c}27.7 \\
1\end{array}$ & $\begin{array}{c}152 . \\
58\end{array}$ & $\begin{array}{c}88.0 \\
9\end{array}$ & 20.9 \\
\hline $\begin{array}{l}\text { Proceedings of the } \\
\text { Annual IEEE } \\
\text { Conference on } \\
\text { Computational } \\
\text { Complexity }\end{array}$ & 99 & 738 & 128 & 93 & 1.43 & $\begin{array}{c}23.8 \\
1\end{array}$ & $\begin{array}{c}2.11 \\
3\end{array}$ & 21 & 6.1 & 7 & $\begin{array}{c}12.1 \\
2\end{array}$ & $\begin{array}{c}51.8 \\
5\end{array}$ & $\begin{array}{c}29.9 \\
3\end{array}$ & $\begin{array}{c}10.3 \\
4\end{array}$ \\
\hline $\begin{array}{c}\text { IEEE } \\
\text { Computational } \\
\text { Intelligence } \\
\end{array}$ & 117 & 769 & 313 & 82 & 3.2 & $\begin{array}{c}16.7 \\
2\end{array}$ & $\begin{array}{c}2.06 \\
6\end{array}$ & 26 & $\begin{array}{c}12.0 \\
4\end{array}$ & 8.67 & $\begin{array}{c}15.0 \\
1\end{array}$ & $\begin{array}{c}90.2 \\
1\end{array}$ & $\begin{array}{c}52.0 \\
8\end{array}$ & $\begin{array}{c}16.9 \\
4\end{array}$ \\
\hline $\begin{array}{l}\text { INFORMS Journal } \\
\text { on Computing }\end{array}$ & 146 & 1682 & 240 & 139 & 1.29 & $\begin{array}{c}28.0 \\
3\end{array}$ & $\begin{array}{c}2.03 \\
4\end{array}$ & 48 & 5 & 16 & $\begin{array}{c}27.7 \\
1\end{array}$ & $\begin{array}{c}107 . \\
33\end{array}$ & $\begin{array}{c}61.9 \\
7\end{array}$ & $\begin{array}{c}13.8 \\
6\end{array}$ \\
\hline $\begin{array}{l}\text { Statistics and } \\
\text { Computing }\end{array}$ & 181 & 3681 & 325 & 177 & 1.71 & $\begin{array}{c}31.1 \\
9\end{array}$ & $\begin{array}{c}1.98 \\
3\end{array}$ & 41 & 7.93 & $\begin{array}{c}13.6 \\
7\end{array}$ & $\begin{array}{c}23.6 \\
7\end{array}$ & $\begin{array}{c}115 . \\
43\end{array}$ & $\begin{array}{c}66.6 \\
5\end{array}$ & $\begin{array}{c}16.8 \\
5\end{array}$ \\
\hline $\begin{array}{l}\text { IEEE Transactions } \\
\text { on Affective } \\
\text { Computing }\end{array}$ & 76 & 2275 & 533 & 70 & 6.79 & $\begin{array}{c}59.8 \\
7\end{array}$ & $\begin{array}{c}1.82 \\
4\end{array}$ & 16 & $\begin{array}{c}33.3 \\
1\end{array}$ & 5.33 & 9.24 & $\begin{array}{c}92.3 \\
5\end{array}$ & $\begin{array}{c}53.3 \\
2\end{array}$ & $\begin{array}{c}22.7 \\
4\end{array}$ \\
\hline $\begin{array}{c}\text { Journal of } \\
\text { Scientific } \\
\text { Computing } \\
\end{array}$ & 303 & 6087 & 613 & 259 & 2.08 & $\begin{array}{c}34.3 \\
9\end{array}$ & $\begin{array}{c}1.77 \\
1\end{array}$ & 42 & 14.6 & 14 & $\begin{array}{c}24.2 \\
5\end{array}$ & $\begin{array}{c}160 . \\
46\end{array}$ & $\begin{array}{c}92.6 \\
4\end{array}$ & 23.9 \\
\hline $\begin{array}{c}\text { International } \\
\text { Journal of Machine } \\
\text { Learning and } \\
\text { Cybernetics }\end{array}$ & 67 & 2330 & 330 & 65 & 4.9 & 35.3 & $\begin{array}{c}1.67 \\
3\end{array}$ & 15 & 22 & 5 & 8.66 & $\begin{array}{c}70.3 \\
6\end{array}$ & $\begin{array}{c}40.6 \\
2\end{array}$ & $\begin{array}{c}17.7 \\
5\end{array}$ \\
\hline $\begin{array}{c}\text { IEEE/ASME } \\
\text { Transactions on } \\
\text { Mechatronics } \\
\end{array}$ & 356 & 5990 & 1738 & 350 & 4.63 & 22.1 & $\begin{array}{c}1.67 \\
1\end{array}$ & 74 & $\begin{array}{c}23.4 \\
9\end{array}$ & $\begin{array}{c}24.6 \\
7\end{array}$ & $\begin{array}{c}42.7 \\
2\end{array}$ & $\begin{array}{c}358 . \\
63\end{array}$ & $\begin{array}{c}207 . \\
05\end{array}$ & $\begin{array}{c}40.7 \\
9\end{array}$ \\
\hline $\begin{array}{l}\text { Automated } \\
\text { Software } \\
\text { Engineering }\end{array}$ & 50 & 1306 & 131 & 40 & 2.46 & $\begin{array}{c}39.5 \\
8\end{array}$ & $\begin{array}{c}1.59 \\
6\end{array}$ & 29 & 4.52 & 9.67 & $\begin{array}{c}16.7 \\
4\end{array}$ & $\begin{array}{c}61.6 \\
4\end{array}$ & $\begin{array}{c}35.5 \\
9\end{array}$ & 10.1 \\
\hline $\begin{array}{l}\text { Computational } \\
\text { Geometry: Theory } \\
\text { and Applications }\end{array}$ & 195 & 1233 & 180 & 141 & 1.21 & 18.4 & $\begin{array}{c}1.41 \\
9\end{array}$ & 35 & 5.14 & $\begin{array}{c}11.6 \\
7\end{array}$ & $\begin{array}{c}20.2 \\
1\end{array}$ & $\begin{array}{c}79.3 \\
7\end{array}$ & $\begin{array}{c}45.8 \\
3\end{array}$ & $\begin{array}{c}12.0 \\
4\end{array}$ \\
\hline $\begin{array}{c}\text { Journal of Graph } \\
\text { Algorithms and } \\
\text { Applications } \\
\end{array}$ & 62 & 779 & 76 & 56 & 1.35 & $\begin{array}{c}24.3 \\
4\end{array}$ & $\begin{array}{c}1.41 \\
7\end{array}$ & 24 & 3.17 & 8 & $\begin{array}{c}13.8 \\
6\end{array}$ & $\begin{array}{c}42.7 \\
1\end{array}$ & $\begin{array}{c}24.6 \\
6\end{array}$ & 7.21 \\
\hline $\begin{array}{c}\text { Advanced } \\
\text { Engineering } \\
\text { Informatics }\end{array}$ & 194 & 2529 & 638 & 177 & 3.58 & $\begin{array}{c}45.9 \\
8\end{array}$ & $\begin{array}{c}1.40 \\
5\end{array}$ & 43 & $\begin{array}{c}14.8 \\
4\end{array}$ & $\begin{array}{c}14.3 \\
3\end{array}$ & $\begin{array}{c}24.8 \\
3\end{array}$ & $\begin{array}{c}165 . \\
63\end{array}$ & $\begin{array}{c}95.6 \\
3\end{array}$ & $\begin{array}{c}24.3 \\
9\end{array}$ \\
\hline $\begin{array}{c}\text { Artificial } \\
\text { Intelligence and } \\
\text { Law }\end{array}$ & 44 & 799 & 65 & 40 & 1.41 & $\begin{array}{c}44.3 \\
9\end{array}$ & 1.39 & 22 & 2.95 & 7.33 & 12.7 & $\begin{array}{c}37.8 \\
2\end{array}$ & $\begin{array}{c}21.8 \\
3\end{array}$ & 6.56 \\
\hline $\begin{array}{c}\text { Theory and } \\
\text { Practice of Logic } \\
\text { Programming } \\
\end{array}$ & 116 & 1301 & 222 & 112 & 2.1 & $\begin{array}{c}28.9 \\
1\end{array}$ & $\begin{array}{c}1.38 \\
5\end{array}$ & 26 & 8.54 & 8.67 & $\begin{array}{c}15.0 \\
1\end{array}$ & $\begin{array}{c}75.9 \\
7\end{array}$ & $\begin{array}{c}43.8 \\
6\end{array}$ & 14 \\
\hline $\begin{array}{c}\text { Fuzzy } \\
\text { Optimization and } \\
\text { Decision Making }\end{array}$ & 66 & 721 & 150 & 63 & 2 & $\begin{array}{c}21.2 \\
1\end{array}$ & $\begin{array}{c}1.37 \\
3\end{array}$ & 29 & 5.17 & 9.67 & $\begin{array}{c}16.7 \\
4\end{array}$ & $\begin{array}{c}65.9 \\
5\end{array}$ & $\begin{array}{c}38.0 \\
8\end{array}$ & 11 \\
\hline $\begin{array}{l}\text { Computers and } \\
\text { Mathematics with } \\
\text { Applications }\end{array}$ & 2108 & $\begin{array}{c}1021 \\
6\end{array}$ & 5600 & 2048 & 2.39 & $\begin{array}{c}28.5 \\
4\end{array}$ & $\begin{array}{c}1.34 \\
3\end{array}$ & 69 & $\begin{array}{c}81.1 \\
6\end{array}$ & 23 & $\begin{array}{c}39.8 \\
4\end{array}$ & $\begin{array}{c}621 . \\
61\end{array}$ & $\begin{array}{c}358 . \\
89\end{array}$ & $\begin{array}{c}74.3 \\
7\end{array}$ \\
\hline $\begin{array}{c}\text { International } \\
\text { Journal of } \\
\text { Artificial } \\
\end{array}$ & 24 & 640 & 62 & 23 & 2.09 & $\begin{array}{c}53.3 \\
3\end{array}$ & $\begin{array}{c}1.32 \\
9\end{array}$ & 10 & 6.2 & 3.33 & 5.77 & 24.9 & $\begin{array}{c}14.3 \\
8\end{array}$ & 7.21 \\
\hline $\begin{array}{c}\text { Journal of } \\
\text { Artificial } \\
\text { Intelligence }\end{array}$ & 160 & 3497 & 495 & 160 & 2.23 & $\begin{array}{c}58.2 \\
8\end{array}$ & $\begin{array}{c}1.31 \\
2\end{array}$ & 76 & 6.51 & $\begin{array}{c}25.3 \\
3\end{array}$ & $\begin{array}{c}43.8 \\
8\end{array}$ & $\begin{array}{c}193 . \\
96\end{array}$ & $\begin{array}{c}111 . \\
98\end{array}$ & $\begin{array}{c}20.4 \\
7\end{array}$ \\
\hline $\begin{array}{l}\text { Mechanism and } \\
\text { Machine Theory }\end{array}$ & 433 & 3757 & 992 & 421 & 2.08 & $\begin{array}{c}25.3 \\
9\end{array}$ & $\begin{array}{c}1.30 \\
3\end{array}$ & 60 & $\begin{array}{c}16.5 \\
3\end{array}$ & 20 & $\begin{array}{c}34.6 \\
4\end{array}$ & $\begin{array}{c}243 . \\
97\end{array}$ & $\begin{array}{c}140 . \\
85\end{array}$ & $\begin{array}{c}30.5 \\
3\end{array}$ \\
\hline $\begin{array}{l}\text { Empirical Software } \\
\text { Engineering }\end{array}$ & 90 & 4046 & 303 & 74 & 3.76 & $\begin{array}{c}51.8 \\
7\end{array}$ & $\begin{array}{c}1.28 \\
5\end{array}$ & 39 & 7.77 & 13 & $\begin{array}{c}22.5 \\
2\end{array}$ & $\begin{array}{c}108 . \\
71\end{array}$ & $\begin{array}{c}62.7 \\
6\end{array}$ & $\begin{array}{c}16.2 \\
5\end{array}$ \\
\hline Algorithmica & 393 & 5117 & 433 & 330 & 1.21 & $\begin{array}{c}26.5 \\
1\end{array}$ & $\begin{array}{c}1.28 \\
3\end{array}$ & 50 & 8.66 & $\begin{array}{c}16.6 \\
7\end{array}$ & $\begin{array}{c}28.8 \\
7\end{array}$ & $\begin{array}{c}147 . \\
14\end{array}$ & $\begin{array}{c}84.9 \\
5\end{array}$ & $\begin{array}{c}19.5 \\
7\end{array}$ \\
\hline $\begin{array}{c}\text { International } \\
\text { Journal of } \\
\text { Intelligent Systems }\end{array}$ & 176 & 1918 & 463 & 169 & 2 & 27.4 & $\begin{array}{c}1.26 \\
8\end{array}$ & 46 & $\begin{array}{c}10.0 \\
7\end{array}$ & $\begin{array}{c}15.3 \\
3\end{array}$ & $\begin{array}{c}26.5 \\
6\end{array}$ & $\begin{array}{c}145 . \\
94\end{array}$ & $\begin{array}{c}84.2 \\
6\end{array}$ & $\begin{array}{c}20.4 \\
2\end{array}$ \\
\hline $\begin{array}{l}\text { IEEE Transactions } \\
\text { on Parallel and } \\
\text { Distributed }\end{array}$ & 558 & 7007 & 1969 & 517 & 3.75 & 31 & $\begin{array}{c}1.25 \\
2\end{array}$ & 78 & $\begin{array}{c}25.2 \\
4\end{array}$ & 26 & $\begin{array}{c}45.0 \\
3\end{array}$ & $\begin{array}{c}391 . \\
9\end{array}$ & $\begin{array}{c}226 \\
26\end{array}$ & $\begin{array}{c}43.4 \\
9\end{array}$ \\
\hline
\end{tabular}




\begin{tabular}{|c|c|c|c|c|c|c|c|c|c|c|c|c|c|c|}
\hline $\begin{array}{c}\text { Control } \\
\text { Engineering } \\
\text { Practice }\end{array}$ & 405 & 5040 & 1372 & 393 & 3.12 & $\begin{array}{c}28.6 \\
4\end{array}$ & $\begin{array}{c}1.24 \\
5\end{array}$ & 67 & $\begin{array}{c}20.4 \\
8\end{array}$ & $\begin{array}{c}22.3 \\
3\end{array}$ & $\begin{array}{c}38.6 \\
8\end{array}$ & $\begin{array}{c}303 \\
19\end{array}$ & $\begin{array}{c}175 \\
05\end{array}$ & $\begin{array}{c}36.1 \\
2\end{array}$ \\
\hline $\begin{array}{c}\text { Artificial } \\
\text { Intelligence } \\
\text { Review }\end{array}$ & 161 & 3956 & 449 & 109 & 2.95 & $\begin{array}{c}51.3 \\
8\end{array}$ & $\begin{array}{c}1.24 \\
2\end{array}$ & 40 & $\begin{array}{c}11.2 \\
3\end{array}$ & $\begin{array}{c}13.3 \\
3\end{array}$ & $\begin{array}{c}23.0 \\
9\end{array}$ & $\begin{array}{c}134 . \\
01\end{array}$ & $\begin{array}{c}77.3 \\
7\end{array}$ & $\begin{array}{c}20.2 \\
2\end{array}$ \\
\hline $\begin{array}{c}\text { Mathematics of } \\
\text { Control, Signals, } \\
\text { and Systems }\end{array}$ & 45 & 908 & 63 & 39 & 1.53 & $\begin{array}{c}31.3 \\
1\end{array}$ & $\begin{array}{c}1.20 \\
6\end{array}$ & 26 & 2.42 & 8.67 & $\begin{array}{c}15.0 \\
1\end{array}$ & $\begin{array}{c}40.4 \\
7\end{array}$ & $\begin{array}{c}23.3 \\
7\end{array}$ & 6.08 \\
\hline $\begin{array}{l}\text { ACM Transactions } \\
\text { on Software } \\
\text { Engineering and }\end{array}$ & 51 & 1771 & 207 & 49 & 4.33 & $\begin{array}{c}49.1 \\
9\end{array}$ & $\begin{array}{c}1.19 \\
7\end{array}$ & 53 & 3.91 & $\begin{array}{c}17.6 \\
7\end{array}$ & 30.6 & $\begin{array}{c}104 . \\
74\end{array}$ & $\begin{array}{c}60.4 \\
7\end{array}$ & $\begin{array}{c}12.4 \\
1\end{array}$ \\
\hline $\begin{array}{c}\text { Engineering } \\
\text { Applications of } \\
\text { Artificial }\end{array}$ & 430 & 8962 & 1336 & 422 & 2.95 & $\begin{array}{c}36.5 \\
8\end{array}$ & $\begin{array}{c}1.18 \\
6\end{array}$ & 54 & $\begin{array}{c}24.7 \\
4\end{array}$ & 18 & $\begin{array}{c}31.1 \\
8\end{array}$ & $\begin{array}{c}268 . \\
6\end{array}$ & $\begin{array}{c}155 . \\
07\end{array}$ & $\begin{array}{c}35.8 \\
1\end{array}$ \\
\hline $\begin{array}{c}\text { Mathematical and } \\
\text { Computer } \\
\text { Modelling } \\
\end{array}$ & 1171 & $\begin{array}{c}1225 \\
7\end{array}$ & 2517 & 1114 & 2.27 & $\begin{array}{c}24.8 \\
1\end{array}$ & $\begin{array}{c}1.15 \\
7\end{array}$ & 59 & $\begin{array}{c}42.6 \\
6\end{array}$ & $\begin{array}{c}19.6 \\
7\end{array}$ & $\begin{array}{c}34.0 \\
6\end{array}$ & $\begin{array}{c}385 \\
36\end{array}$ & $\begin{array}{c}222 \\
49\end{array}$ & $\begin{array}{c}49.5 \\
8\end{array}$ \\
\hline $\begin{array}{c}\text { Integrated } \\
\text { Computer-Aided } \\
\text { Engineering } \\
\end{array}$ & 89 & 1276 & 327 & 81 & 4.3 & $\begin{array}{c}45.5 \\
7\end{array}$ & $\begin{array}{c}1.11 \\
9\end{array}$ & 25 & $\begin{array}{c}13.0 \\
8\end{array}$ & 8.33 & $\begin{array}{c}14.4 \\
3\end{array}$ & $\begin{array}{c}90.4 \\
2\end{array}$ & 52.2 & $\begin{array}{c}17.3 \\
8\end{array}$ \\
\hline $\begin{array}{c}\text { Artificial } \\
\text { Intelligence in } \\
\text { Medicine }\end{array}$ & 172 & 2487 & 426 & 158 & 2.35 & $\begin{array}{c}40.7 \\
7\end{array}$ & $\begin{array}{c}0.86 \\
5\end{array}$ & 53 & 8.04 & $\begin{array}{c}17.6 \\
7\end{array}$ & 30.6 & $\begin{array}{c}150 \\
26\end{array}$ & $\begin{array}{c}86.7 \\
5\end{array}$ & $\begin{array}{c}19.3 \\
1\end{array}$ \\
\hline $\begin{array}{c}\text { Advances in } \\
\text { Engineering } \\
\text { Software }\end{array}$ & 342 & 372 & 760 & 332 & 2.23 & 46.5 & 0.85 & 39 & $\begin{array}{c}19.4 \\
9\end{array}$ & 13 & $\begin{array}{c}22.5 \\
2\end{array}$ & $\begin{array}{c}172 \\
16\end{array}$ & 99.4 & $\begin{array}{c}26.8 \\
5\end{array}$ \\
\hline
\end{tabular}

where,

TD3: Total Docs.; TC3: Total Cites; CD3: Citable Docs.; CD2: Cites / Doc.; RD: Ref. / Doc.; SJR: SciJournal Rank

\section{RESULTS AND DISCUSSION}

Regression analysis implemented to assess the improvement of several index values for all computer science journals and the objective is to identify the important citation parameters and how they behave in a dataset. This is based on the fact that a high index value attracts more number of papers to that particular journal. The outcome of the program is prediction of dependent variables and several validation parameters are analysed such as $\mathrm{r} 2$, adjusted $\mathrm{r} 2$, F-statistic etc. The obtained equations were given below.

h-index $=0.0858 *$ TD $3-0.0001 *$ TR $3+$

$0.0268 * \mathrm{TC} 3-0.1234 * \mathrm{CD} 3-0.5905 * \mathrm{CD} 2-$

$0.1207 * \mathrm{RD}+3.2732 * \mathrm{SJR}+40.6617$

$\mathrm{r} 2=0.632$, adjusted $\mathrm{r} 2=0.613$,

F-statistic $=32.90$

(Eq.1)

a-index $=\quad-0.0034 * \mathrm{TD} 3+0.0009 * \mathrm{TR} 3-$

$0.0026 * \mathrm{TC} 3+0.0324 * \mathrm{CD} 3+3.1280 * \mathrm{CD} 2-$

\section{$0.0581 * \mathrm{RD}-0.5664 * \mathrm{SJR}-0.4094$}

$\mathrm{r} 2=0.718$, adjusted $\mathrm{r} 2=0.703$,

F-statistic $=48.78$

$\mathrm{m}$-index $=0.0286 * \mathrm{TD} 3-0.0003 * \mathrm{TR} 3+$

$0.0089 * \mathrm{TC} 3-0.0411 * \mathrm{CD} 3-0.1969 * \mathrm{CD} 2-$ $0.0403 * \mathrm{RD}+1.0916 * \mathrm{SJR}+13.5543$

$\mathrm{r} 2=0.632$, adjusted $\mathrm{r} 2=0.613$,

F-statistic $=32.90$

(Eq.3)

q2-index $=0.0495 * \mathrm{TD} 3-0.0006 * \mathrm{TR} 3+$

$0.0155 * \mathrm{TC} 3-0.0712 * \mathrm{CD} 3-0.3406 * \mathrm{CD} 2-$ $0.0697 * \mathrm{RD}+1.8895 * \mathrm{SJR}+23.4749$

$\mathrm{r} 2=0.632$, adjusted $\mathrm{r} 2=0.613$,
F-statistic $=32.90$

(Eq.4)

r-index $=0.2652 * \mathrm{TD} 3+0.0007 * \mathrm{TR} 3+$

$0.1640 * \mathrm{TC} 3-0.3636 * \mathrm{CD} 3+0.0598 * \mathrm{CD} 2-$ $0.4406 * \mathrm{RD}+8.7870 * \mathrm{SJR}+84.0066$

$\mathrm{r} 2=0.936$, adjusted $\mathrm{r} 2=0.932$,

F-statistic $=278.3$

a-r index $=0.1531 * \mathrm{TD} 3+0.0004 * \mathrm{TR} 3+$

$0.0947 * \mathrm{TC} 3-0.2099 * \mathrm{CD} 3+0.0344 * \mathrm{CD} 2-$ $0.2544 * \mathrm{RD}+5.0732 * \mathrm{SJR}+48.5020$

$\mathrm{r} 2=0.936$, adjusted $\mathrm{r} 2=0.932$,

F-statistic $=278.3$

(Eq.6)

e-index $=0.0272 * \mathrm{TD} 3+0.0007 * \mathrm{TR} 3+$

$0.0067 * \mathrm{TC} 3-0.0167 * \mathrm{CD} 3+1.5634 * \mathrm{CD} 2-$ $0.0656 * \mathrm{RD}+0.1544 * \mathrm{SJR}+10.6136$

$\mathrm{r} 2=0.962$, adjusted $\mathrm{r} 2=0.960$,

F-statistic $=478.3$

(Eq.7)

Considering h-index as dependent variable, from the linear regression analysis it is observed that an increase in SJR, TD3 and TC 3 with a decrease in remaining parameters is essential for a high h-index value. It is understood that in all cases except a-index, an increase in SJR value is suggested which means that SJR contributes positively to enhance index factor of journals. A similar observation was made with TD3, except a-index, this parameter showed a nominal increase in this value might favour better index values. From equation-2, it is evidenced that $\mathrm{CD} 2$ should be increased to more extent when compared to remaining parameters to produce high a-index. Moreover, decrease in RD in all cases suggest that RD has negative effect on all indices. Therefore a decrease in references per document would favour better index values. Overall data on TC3 suggested that this parameter is not of much importance. Finally it can be stated that all computer science journal selected in the study emphasized the role of citation parameters to increase index values. In all cases, a 
negative value represents that parameter value should be reduced in order to gain increase in a particular value and a positive contribution to index value is ascertained for positive coefficient values

\section{CONCLUSION}

In this paper, an attempt has been made to study the relationship between citation parameters such as Total Docs.; Total Cites; Citable Docs.; Cites / Doc.; Ref. / Doc.; SciJournal Rank etc., and bibliometric indices such as hindex, a-index, m-index, q2 index, r-index, a-r index and eindex respectively. A linear regression program written in python suggested that an increase in TD3 and SJR for all computer science journals would enhance index values except a-index. Therefore, considering the positivity and negative contribution of citation parameters on journals is deemed necessary to increase likelihood of paper submission to journals.

\section{REFERENCES}

[1] E. Garfield, "Citation analysis as a tool in journal evaluation”, Science, vol. 178, pp. 471-479, 1972

[2] J. D. West, T. C. Bergstrom, C. T. Bergstrom, "The Eigenfactor Metrics: A network approach to assessing scholarly journals," College of Research Libraries, vol. 71, pp. 236-244, 2010.

[3] B. Gonzalez-Pereira, V. P. Guerrero-Bote, F. MoyaAnegon, "A new approach to the metric of journals scientific prestige: The SJR indicator," Journal of Informetrics vol 4, pp. 379-391, 2010.

[4] Joshi MA Bibliometric indicators for evaluating the quality of scientific publications. J Contemp Dent Pract. 2014 Mar 1;15(2):258-62.

[5] http://www.vub.ac.be/BIBLIO/itp/lecturers/ronald_rouss eau/ronald_roussea_stim1_bibliometrics_russell.pdf
[6] Hirsch, J.E. (2005) an index to quantify an individual's scientific research output. Proceedings of the National Academy of Sciences 102:16569-16572.

[7] Olden, J.D. (2007) how do ecological journals stack-up? Ranking of scientific quality according to the h-index. Eco science 14(3):370-376.

[8] Rousseau, R. (2007) The influence of missing publications on the Hirsch index. Journal of Informetrics $1: 2-7$.

[9] Vanclay, J.K. (2007) On the robustness of the h-index. Journal of the American Society for Information Science and Technology 58(10):1547-1550.

[10] Steven B. Bird. Journal Impact Factors, h Indices, and Citation Analyses in Toxicology. JOURNAL OF MEDICAL TOXICOLOGY 2008, 4(4): 261-274.

[11] Egghe, L. (2006). Theory and practice of the g-index. Scientometrics, 69(1), 131-152.

[12] Kosmulski, M. (2006). A new Hirsch-type index saves time and works equally well as the original h-index. ISSI Newsletter, 2(3), 4-6.

[13] Rousseau, R. (2007). The influence of missing publications on the Hirsch index. Journal of Informetrics, $1(1), 2-7$.

[14] Jin, B. (2007). The AR-index: complementing the hindex. ISSI Newsletter, 3(1), 6.

[15] Bornmann, L., Mutz, R., \& Daniel, H. (2008). Are there better indices for evaluation purposes than the h-index? A comparison of nine different variants of the h-index using data from biomedicine. Journal of the American Society for Information Science and Technology, 59(5), 830-837.

[16] http://www.scimagojr.com/journalrank.php

[17] http://www.elsevier.com. 